\title{
Cancrena o gangrena (o cangrena) per me pari sono
}

\author{
Paolo D'Achille
}

PUBBLICATO: 06 DICEMBRE 2019

\section{Quesito:}

Sono arrivate varie domande sull'alternativa tra cancrena e gangrena. A molti lettori la forma cancrena risulta quella più comune, ma alcuni rilevano la presenza di gangrena nel linguaggio della medicina. C'è chi segnala anche la variante cangrena e chi chiede l'etimologia della parola.

\section{Cancrena o gangrena (o cangrena) per me pari sono}

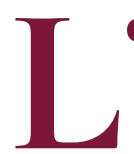

9 italiano è una lingua tra le più ricche sia di polimorfi, cioè di forme equivalenti che presentano differenze sul piano fonetico e/o morfologico (come olivo e ulivo, pronuncia e pronunzia, devo e debbo, ecc.), sia anche di allòtropi, cioè di parole che, pur dipendendo da una stessa base (in genere una parola latina), hanno assunto forma e significato diversi, perché l'una è entrata nel nostro lessico per trafila diretta (quindi attraverso il latino volgare), l'altra è stata recuperata per via dotta; quest'ultima, in genere, è meno antica della prima e si è mantenuta più fedele sul piano sia formale sia semantico alla base.

Tra le tante coppie di parole che si possono citare ci limitiamo a ricordare pieve (popolare) e plebe (dotta), entrambe dal lat. plebem; ristorare (popolare) e restaurare (dotta), dal lat. restaurare; vezzo (popolare) e vizio (dotta), dal lat. vitium. A volte gli allòtropi sono più di due, perché alla parola dotta si affianca più di un esito popolare: per esempio, raggio e razzo sono ambedue derivati dal lat. radium, da cui deriva anche (seppure attraverso il francese) radio, nome dato all'elemento chimico scoperto da Maria Curie perché emette radiazioni.

La stessa cosa vale per i verbi (che abbiamo citato in una risposta recente) coagulare, cagliare e quagliare, tutti e tre dal lat. coagulare, e, ancora, per i tre derivati da fabulam, e cioè favola, fiaba e fola. Come è avvenuto in alcuni dei casi appena indicati, la formazione di allòtropi è talvolta dovuta al "prestito interno": parole che l'italiano ha tratto da varietà dialettali diverse da quella toscana si sono aggiunte alla variante toscana, mantenendo o sviluppando un diverso significato, o assumendo una diversa categoria grammaticale (è il caso di potere e podere, sacra e sagra, pippione e piccione); in altri casi ancora a produrre l'allotropia è stato l'ingresso di parole straniere: derivano dal lat. caput sia capo, sia attraverso il francese - l'antico ceffo e il più moderno chef; accanto al vecchio solaio, tramite l'inglese abbiamo recuperato anche, con diverso significato, il lat. solarium che ne è alla base.

Rientra tra gli allòtropi anche la coppia segnalata dai nostri lettori, cancrena e gangrena, che però presenta una particolarità: le due parole hanno infatti lo stesso significato, indicano la stessa cosa e cioè, per riportare la definizione che di cancrena dà il GRADIT, la "distruzione e morte di un tessuto o di una parte del corpo, spec. di un arto, per mancato apporto vascolare o grave ostacolo al deflusso del sangue". Lo stesso dizionario, accanto a questa accezione, etichettata come propria del lessico medico, segnala quella, botanica, di "marciume" e anche quella, figurata, di "vizio, corruzione diffusa", mentre registragangrena come semplice variante dicancrena. Da cancrena derivano l'aggettivo cancrenoso (o gangrenoso) e il verbo parasintetico incancrenire (la variante ingangrenire, registrata nel GDLI, è molto rara), che ha sia il senso proprio di 'andare in cancrena', sia quello figurato di 'divenire più grave e radicato' (riferito a vizi, fatti negativi, ecc.). 
Per capire le ragioni di questa allotropia, e per cercare di individuare la parola dotta, dobbiamo guardare all'etimologia. Alla base delle nostre voci c'è il termine lat. gangraenam, a sua volta dal gr. gángraina, derivato del verbo gráó 'divorare'; come segnala l'Etimologico, dalla stessa base derivano il fr. gangréne e lo sp. gangrena. Dunque, la $g$ che troviamo all'inizio e all'interno della forma gangrena è etimologica e non popolare, come pure avrebbe potuto essere. Infatti, dal punto di vista fonetico, la giniziale rappresenta a volte la sonorizzazione di una velare sorda iniziale latina, che è un tratto caratteristico dell'area toscana e centrale (si pensi a forme come gastigo, gastigare per castigo, castigare), presente anche in parole dello standard come gatto (dal lat. cattum) o grotta (dal lat. cryptam; cripta è l'allòtropo dotto). A sua volta la $g$ interna potrebbe spiegarsi in area toscana come assimilazione alla $g$ - iniziale oppure, in area centromeridionale, con la lenizione dopo nasale tipica dei dialetti a sud della linea Roma-Ancona (che potrebbe spiegare anche la forma cangrena, citata da un lettore). Ma non è così gangrena è la forma più vicina alla base latina e cangrena (che ha come vedremo, varie attestazioni, nel passato come nel presente) pare una forma "di compromesso" tra gangrena e cancrena. È proprio la forma cancrena, dunque, che richiede di essere spiegata.

Ma la spiegazione è piuttosto semplice, tanto che i dizionari etimologici sono tutti concordi nel sostenere l'influsso di un altro termine della medicina, a cui la parola è stata accostata: cancro 'tumore maligno', a sua volta cosi detto per la forma delle ramificazioni, che ricordano le chele del cancro, cioè del granchio (dal lat. cancrum: ennesima coppia di allotropi!). Abbiamo dunque un caso di "incrocio etimologico", un esempio di etimologia popolare o paretimologia (anche se, personalmente, non escluderei del tutto la possibilità che ci sia stata anche una spinta "ipercorrettistica" a favorire la desonorizzazione delle due $g$ ).

Cancrena, dunque, è l'allòtropo formatosi per via popolare, mentre gangrena è la variante dotta. Ce lo confermano le più antiche attestazioni delle due forme: cancrena è documentato già nel Trecento (in due autori settentrionali: cfr. TLIO), mentre i primi esempi di gangrena sono posteriori: il più antico riportato dal GDLI, individuabile grazie alla possibilità di consultare questo monumentale dizionario storico dagli scaffali elettronici del sito della nostra Accademia è nella Cirugia universale e perfetta di Giovanni Andrea Dalla Croce (Venezia, 1583) e fa esplicito riferimento al greco: "La cancrena, o gangrena, che così la chiamano i Greci, [...] o mortificazione, come dicono i Latini, è una alterazione morticina della parte cutanea".

Per quanto riguarda la frequenza d'uso, lo stesso GDLI, tra definizioni ed esempi, di gangrena offre I7 attestazioni al singolare e 5 al plurale (quasi sempre in testi di medicina), mentre di cancrena (la voce messa a lemma) gli esempi sono ben più numerosi: i26 al singolare e 30 al plurale. La variante cangrena, infine, è documentata nel GDLI 5 volte al singolare e 4 al plurale (un esempio è del Baretti).

Il corpus MIDIA restituisce I2 occorrenze (distribuite dal sec. XV al XX) di cancrena, solo 2 (ottonovecentesche) di gangrena e nessuna di cangrena. Nel PTLLIN abbiamo i4 esempi in I2 opere di cancrena/e e nessuno di gangrena né di cangrena. In Internet, invece, una ricerca tramite Google effettuata il 24 agosto 2019 dà 24I.00o risultati per cancrena, 84.000 per cangrena e ben 2.I20.00o per gangrena, tra cui la voce di Wikipedia, che inizia cosi: "La gangrena, nel linguaggio comune detta cancrena, è un tipo di necrosi tissutale causata generalmente da carente apporto sanguigno della parte interessata".

Evidentemente, la preferenza per il termine considerato più tecnico in quanto adottato nel linguaggio medico (che, come è noto, predilige i grecismi) e anche (e direi soprattutto) il modello dell'inglese gangrene (che questa lingua ha preso dal fr. gangréne) stanno determinando un ribaltamento nei rapporti di forza tra le varianti, che sono comunque da considerare tutte corrette. Si può solo 
suggerire di evitare la forma cangrena (sempre minoritaria, e non registrata nei dizionari sincronici) e di usare cancrena e non gangrena nel significato figurato, che si è prodotto a partire dalla variante popolare.

\section{Cita come:}

Paolo D'Achille, Cancrena o gangrena (o cangrena) per me pari sono, "Italiano digitale", 2019, XI, 2019/4 (ottobre-dicembre)

DOI: $10.35948 / 2532-9006 / 2020.3248$

\section{Copyright 2019 Accademia della Crusca}

Pubblicato con licenza creative commons CC BY-NC-ND 